\title{
Photonuclear Effect on ITER Operation
}

\author{
Farshid Tabbakh, Amir Hossein Gharashi \\ Plasma and Nuclear Fusion, Nuclear Science and Technology, Tehran, Iran \\ Email: ftabbakh2000@yahoo.com
}

Received 3 June 2015; accepted 13 October 2015; published 20 October 2015

\begin{abstract}
In this study, neutron and gamma radiation produced during the ITER tokamak operation including the nuclear heating were studied. This is an approach for analyzing the neutron flux by their contributions. The calculations were conducted using Geant4 Monte Carlo method based on the ITER simplified geometry. It was found that for present design of ITER, only $0.1 \%$ of neutrons is contributed by photonuclear interaction while, $33 \%$ belongs to the $(\mathrm{n}, \mathrm{xn})$ reaction. Finally by adding Beryllium element in the coolant leading to the increase in the neutron flux by increasing the rate of $(\gamma, \mathbf{n})$ reactions was calculated.
\end{abstract}

\section{Keywords}

ITER, Photo-Nuclear, Wall-Loading, Energy Deposition, GEANT4

\section{Introduction}

The most promising candidate for future power generation is fusion energy produced in tokamaks by means of D-D and D-T reactions. In this study, International Thermonuclear Experimental Reactor (ITER) [1] [2] is simulated in nuclear points of view to analyze theneutron flux and the total deposited energy.

To this purpose, Monte Carlo method as the most reliable tools has been used. One of the most powerful Monte Carlo tools are MCNPX and GEANT4 and due to the rare use of GEANT4 in the tokamak calculation, this method has been verified in this work to present the reliability of GEANT4 in such calculations.

First, the GEANT4 results were verified using the ITER available data and then, the increase in nuclear heating was investigated for modification in cooling water by means of adding berylliumto increase the $(\gamma, n)$ reactions.

The calculation was conducted using GEANT 4.9.4 [3] with simplified geometry of ITER [4] [5].

\section{Calculation and Methods}

The simulation of ITER in this work was conducted based on the simple geometry, materials presented in [4]-[6]. There are three neutron sources in tokamaks, the plasma as the main source and two reactions of (n, xn) and $(\gamma$, $\mathrm{n})$. It was shown that though, the photo-neutrons population are negligible but play important rule in case of using proper materials [7]. In this case one can see that the neutron population and energy deposition will found a large increase leading to efficiency enhancement up to several times more. The nuclear heating (energy deposition) was calculated for the cases of pure water and Beryllium added to water to show the importance of increasing the photonuclear interactions. For verifying the GEANT4 results, the neutron wall loading and the neutron flux in (FW) was calculated and compared to references [4] and [5]. 
In GEANT4, the $(\gamma, \mathrm{n})$ reaction should be included in the "Physiclist" by selecting the proper physics and model [8]. Because of the large dimensions of ITER reactor, the simulations were performed for $10^{8}$ events to obtain the best score as much as possible. Our calculations conducted based on theGEANT4.9.4, G4NDL.3.14 as the neutron data library, CHIPS model for photonuclear and EMLOW.6.19 as EM interactions.

The geometry, materials and the related compounds are based on the information reported in references [4] [5] that, the main parameters are presented in Table 1. As it was mentioned, the geometry in this work considered as a simplified geometry due to the complicated process of translating the CAD to GEANT4. This geometry is presented in Figure 1.

The source in our simulations was defined as a volumetric donate shape source which emits $14.1 \mathrm{MeV}$ neutrons isotropically. Finally, the importance of the photonuclear interactions was investigated by adding Beryllium in the coolant as an extra neutron source to find the changes in the total energy deposition in Blanket and Vacuum vessel (VV) fillings. Considering $(\gamma, \mathrm{n})$ reaction threshold of 1.6 MeV [9] [10] for Beryllium, the gamma-rays with energies more than threshold can be converted to photo-neutrons and consequently increasing the deposited energy. It will lead to an increase in the thermal power of the reactor and the efficiency as well.

\section{Results and Discussions}

The following results present the neutron distribution generated in ITER simplified geometry. Figure 2 illustrates the fluxes versus the distance from plasma center around the vacuum vessel filling. The red line represents the real neutron flux which includes $(\gamma, \mathrm{n})$ reaction and black line represents the neutron flux if there were no photo-neutrons. To obtain the results of "without photo-neutron" (black line) the photonuclear reactions were switched off by removing the CHIPS model from the Physics List. Thus, the neutrons detected in the results were all from any reaction except the photonuclear. The calculation depicts a negligible contribution near only $0.1 \%$ of the neutrons population come from the photonuclear while, according to the additional GEANT4 output data, around 33\% of the neutrons were produced from (n, xn) interaction as another source of neutrons.

Figure 3 shows the differences between the two gamma fluxes illustrated by with and without photonuclear corresponding to the red line and black lines respectively at the same distance of Figure 2. As one can see there is a negligible contribution in gamma flux standing for photonuclear included.

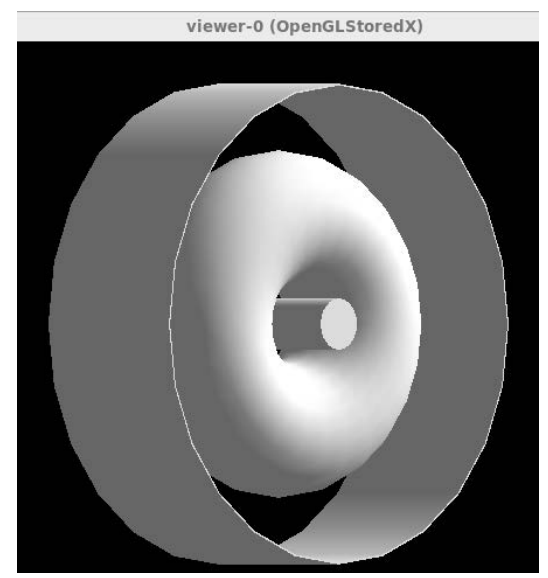

Figure 1. The GEANT4 released geometry drown by OpenGL visualizer.

Table 1. The main parameters of ITER [4] [5].

\begin{tabular}{|c|c|c|}
\hline Parameters & Unit & Value \\
\hline ITER major radius & (m) & 6.21 \\
\hline ITER minor radius & (m) & 2 \\
\hline Neutron emission rate & $\left(\mathrm{s}^{-1}\right)$ & $1.44 \times 10^{20}$ \\
\hline Av. neutron wall loading & $\left(\mathrm{MW} / \mathrm{m}^{2}\right)$ & 0.57 \\
\hline
\end{tabular}


Figure 4 shows the neutron spectra in the first wall (FW) at distance of $850 \mathrm{~cm}$ from tokamak center for both with and without $(\gamma, \mathrm{n})$ represented by black line and red line respectively. This figure verify the calculation of nuclear aspects using GEANT4 referring to the same parameter presented in [5].

The average neutron wall-loading over the inner surface of the first wall (FW) can be obtained from the expression $\sum_{i}(f l u x(E i) \times E i)$, In which, flux $(E i)$ is the neutron flux corresponding to the $i^{\text {th }}$ energy bin. Accordingly, the related value of the above expression is $5.38 \times 10^{14} \mathrm{MeV} / \mathrm{cm}^{2} \mathrm{~s}$ and consequently, the neutron wall-loads will be $0.862 \mathrm{MW} / \mathrm{m}^{2}$. The uncertainty between the two conceptual and the detailed geometries causesthe small difference between the results of Monte Carlo methods and the wall loading presented in Table 1.

Figure 5 depicts the importance of gamma to neutron conversion via photonuclear interaction. Inserting Beryllium in the coolant ( can be done using Nano particles suspension) causes an large increase in the total energy deposition in takamak components, the blanket and VV filling up to 3 times.

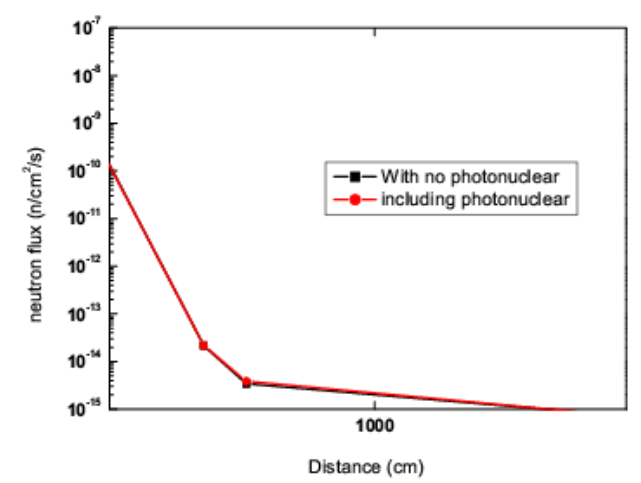

Figure 2. Neutron flux versus the distance around the VV filling.

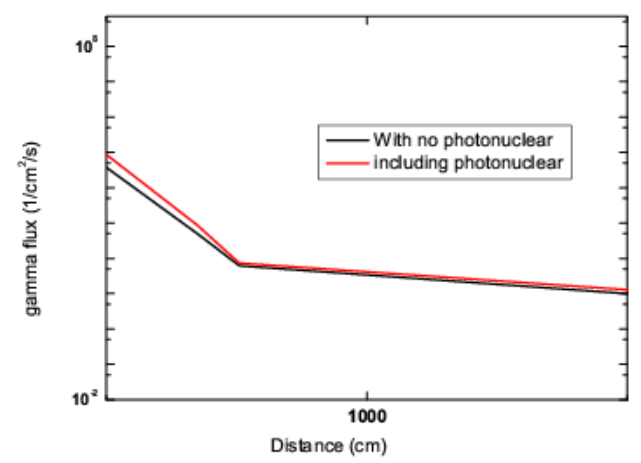

Figure 3. Gamma flux versus the distance around the VV filling.

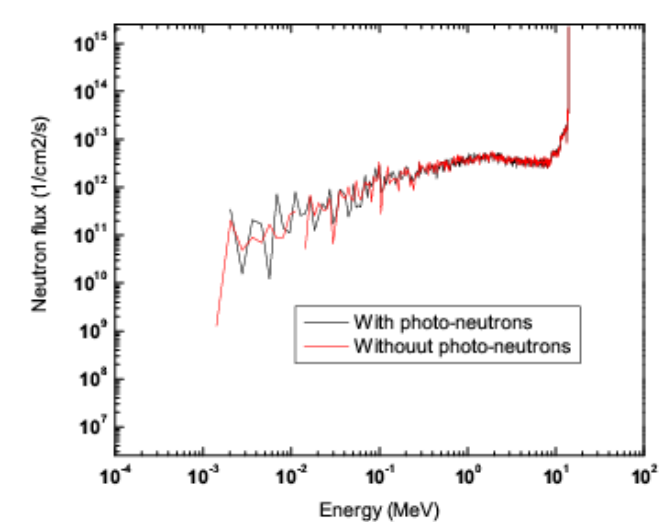

Figure 4. Neutron spectra at the inner surface of FW. 


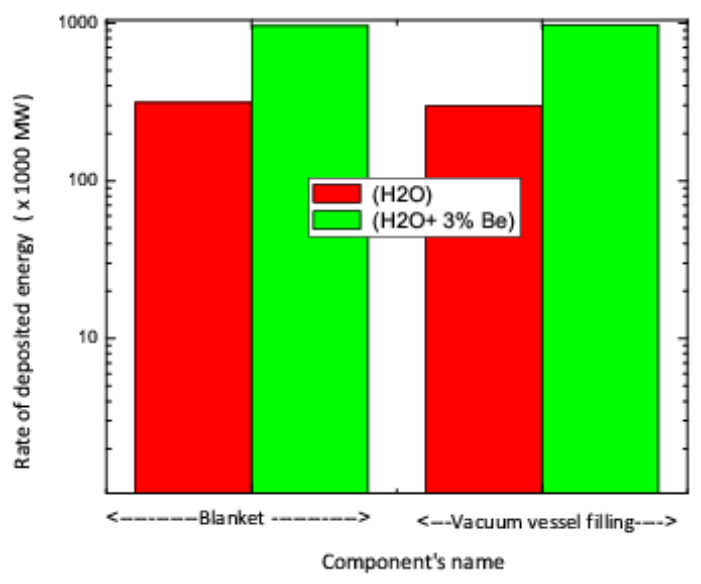

Figure 5. Energy deposition in Blanket and VV filling.

\section{Conclusion}

ITER in this work was studied in the aspects of the contribution of photonuclear interaction and using Geant4 Monte Carlo code. The contribution of produced photo-neutrons via $(\gamma, \mathrm{n})$ reaction in neutron flux was found about $0.1 \%$. The photo-neutrons as a negligible source of neutrons in tokamak can play important roles if they could be increased [7]. Using the proper materials, especially in the coolant to increase the photo-neutrons which leads to an increase in the output power. We found that the efficiency of the system can be enhanced up to several times more than present design of ITER.

\section{References}

[1] ITER Project. http://www.iter.org

[2] ITER Documentation Series (2001) Summary of the ITER Final Design Report IAEA/ITER EDA/DA/22. Vienna/ IAEA.

[3] Geant4 Collaboration (2009) Introduction to GEANT4 User Documentation Version 4.9.4.

[4] Araújo, A., Pereira, C., Veloso, M.A.F. and Costa, A.L. (2010) Flux and Dose Rate Evaluation of ITER System Using MCNP5 Brazilian. Journal of Physics, 40, 58-62. http://dx.doi.org/10.1590/S0103-97332010000100010

[5] Velasquez, C.E., Pereira, C., Veloso, M.A.F. and Costa, A.L. (2014) Modelling Effects on Axial Neutron Flux in a Tokamak Device. Progress in Nuclear Energy, 78, 388-395. http://dx.doi.org/10.1016/j.pnucene.2014.03.005

[6] ITER Documentation Series (1991) ITER Conceptual Design Report IAEA/ITER/DS/18. Vienna/IAEA.

[7] Tabbakh, F. (2015) An Analytical Study of Photonuclear Interaction in ITER Operation for Enhancing the Nuclear Heating. Journal of Fusion Energy, 1-4. http://dx.doi.org/10.1007/s10894-015-9966-8

[8] Geant4 Collaboration (2010) Physics Reference Manual.

[9] IAEA (2002) Photonuclear Data, LA-UR-02-124.

[10] IAEA (2000) TECDOC-1178: Handbook of Photonuclear Data for Applications. Vienna/IAEA. 